\title{
Experience with Contemporary Tip-Edge plus Technique-A Case Report.
}

\author{
Dr. Pravinkumar S. Marure, Dr. Sumitra Reddy, Dr. Suresh Kangane, \\ Dr. Anand Ambekar, Dr. Yatish Joshi \\ Assistant Professor, Department of orthodontics MIDSR dental college and hospital Latur, Maharashtra, India \\ Professor and Head, Department of orthodontics KLE'S institute of dental sciences Bangalore, Karnataka, \\ India. \\ Professor, Department Of Orthodontics MIDSR Dental College and Hospital, Latur. Maharashtra. India. \\ Reader, Department Of Orthodontics MIDSR Dental College and Hospital, Latur. Maharashtra. India. \\ Assistant Professor, Department Of Orthodontics MIDSR Dental College and Hospital, Latur. Maharashtra. \\ India.
}

\begin{abstract}
Aim of this case report was to analyze the treatment mechanics of Tip-Edge Plus appliance. A recent modification to the Tip-Edge bracket system now enables torque and tip corrections to be achieved without the use of auxiliary springs (Side-Winders). Tip Edge Plus employ nickel titanium for root uprighting by using the additional slot of the bracket. The Aesthetics and efficiency in Stage III of Tip Edge Plus are far superior. Tip Edge Plus Appliance is easier to keep clean without auxiliary springs, more comfortable without the added profile of Side-Winders.
\end{abstract}

Key words: Tip-Edge Plus, Side-Winders, Root uprighting.

\section{Introduction:}

The Tip-Edge bracket was invented by Dr P. C. Kesling in 1986, following an analysis of tooth movements occurring during Begg treatment. Kesling determined that it was necessary for each tooth to tip either mesially or distally, but not in both directions. He found this to be true in the correction of all malocclusions whether they were treated with extractions or not and that all the teeth mesial to the extraction site tip distally whilst those distal to it tip mesially. Kesling designed the Tip-Edge ${ }^{\circledR}$ bracket to provide all the benefits of differential tooth movement together with the control required to deliver a predetermined amount of tip and torque as found in pre-adjusted Edgewise systems. He appreciated the ease of manipulation that the edgewise slot conveyed so modified it by removing wedges from two opposite corners of the bracket slot to allow each tooth to tip freely, by a predetermined amount, in one direction and prevent them from tipping in the other direction by the horizontal surfaces of the slot. ${ }^{1-3}$ The Tip-Edge ${ }^{\circledR}$ represented a major advance over the Begg system in terms of patient comfort and ease of handling, utilizing the convenience of labially inserted archwires with conventional tie-wings and elastomerics, in place of gingivally inserted wires retained with brass pins. However, one of the greatest disincentives to using Tip-Edge has been that each bracket requires an auxiliary spring (Side-Winder) to deliver its final prescription. ${ }^{4}$

Special TIP EDGE PLUS brackets (Fig. 1), were prepared to eliminate the use of auxiliary springs in the final root-uprighting phase of treatment (Stage III) that utilized 'deep grooves' throughout, consisting of an auxiliary archwire slot, cut deep to the main arch slot, into which a superelastic wire could readily be fitted to achieve the desired mesiodistal uprighting. When a light flexible wire is passed through the tunnel, a secondorder uprighting force is generated by the deflection of the wire, comparable in degree to that produced by a Side- Winder in conventional Tip-Edge. The auxiliary wire is generally threaded from the midline, in reverse curvature, right side to left, and vice versa. Once the auxiliary wire is in place, the $0.021 \mathrm{X} 0.028$ - inch main archwire is prepared and fitted in exactly the same way as with existing Tip-Edge, retained with elastomeric ligatures. I would like to present my early case treated with new Tip-Edge Plus appliance. ${ }^{4-6}$

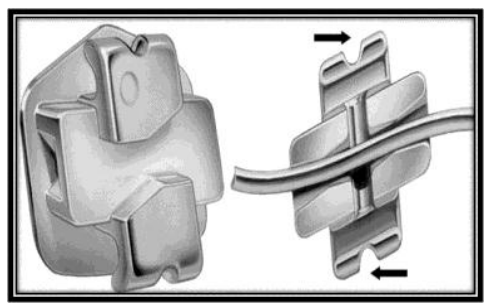

Fig. 1 Tip-Edge Plus Bracket and its deep tunnel running beneath the main arch wire slot. 


\section{DIAGNOSIS AND TREATMENT PLANNING:}

\section{Clinical Case:}

A male patient aged 17 years 5 months presented with an overjet and overbite of $2 \mathrm{~mm}$ and bimaxillary proclination. Cephalometric evaluation revealed a skeletal Class II Malocclusion with angles class I molar relationship with receding chin and a high mandibular plane angle (Fig. 2). The periodontium and the temporoMandibular joints were healthy. The patient requested treatment to improve his dental and facial appearance. To achieve an ideal result, a treatment plan using chin advancement surgery and camouflage orthodontic treatment was proposed. The Patient firmly declined chin advancement surgery, and a treatment was planned around the extraction of four first premolars.
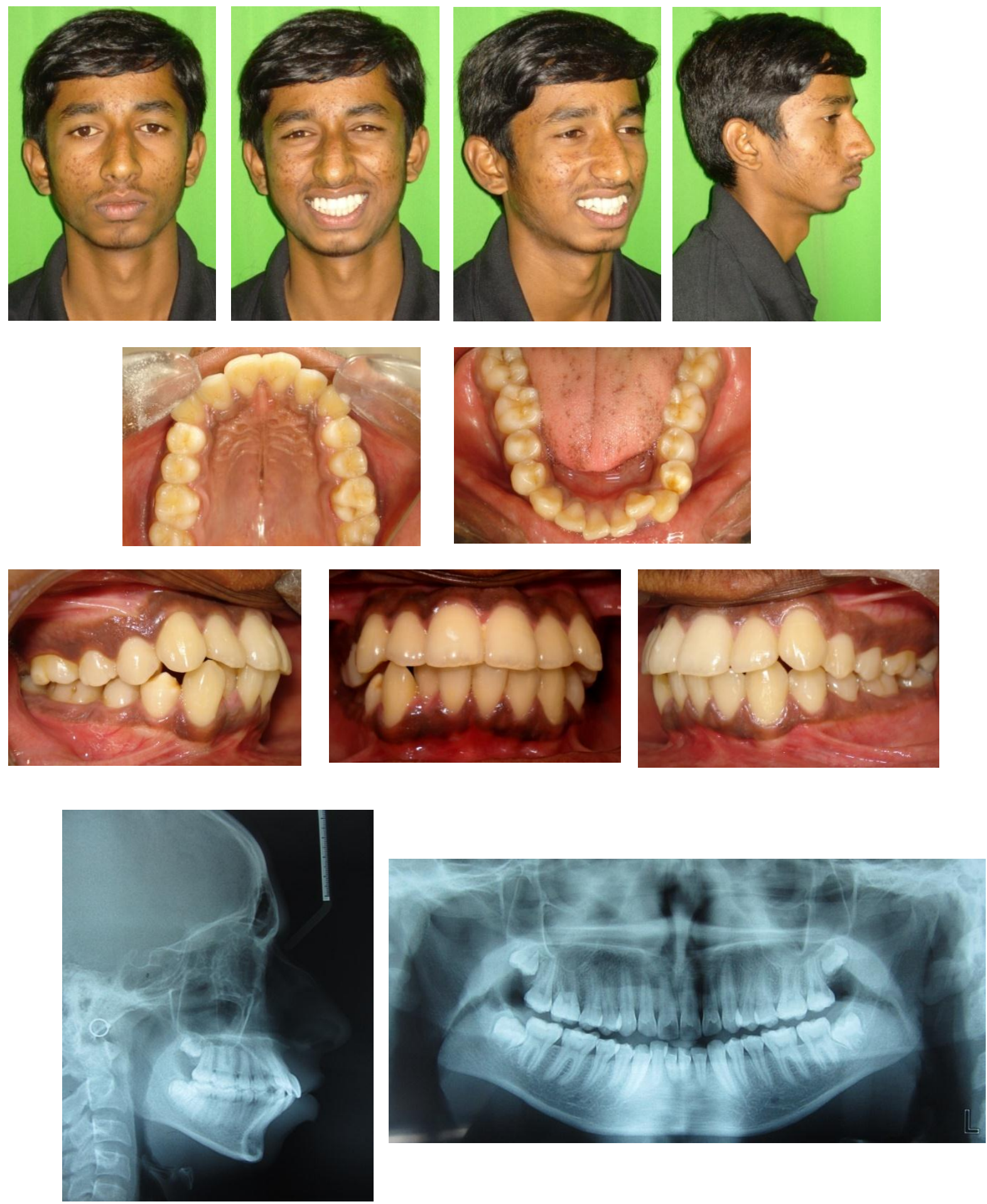

Fig. 2. Pre-treatment extra-oral and intra-oral photographs, lateral cephalograms and panoramin radiograph. 
Experience with Contemporary Tip-Edge Plus Technique-A Case Report.

\section{TREATMENT PROGRESS AND RESULTS:}

Orthodontic treatment started with Tip-Edge Plus appliance. Stage I begins, using high-tensile 0.016inch main archwires with bite-opening 'anchorage bends' and very light Class II elastic traction (0.25-inch, 3.5oz) (Figure 3.) and stage II Five months into treatment, the remaining spaces was closed with 0.020 -inch stainless archwires and intra-maxillary Elastomerics (Figure 4.) were accomplished in the established manner, with no anchorage reinforcement required, apart from very light Class II intermaxillary elastics. Overjet reduction, arch alignment, overbite reduction and space closure were thus achieved in 10 months. There was a torque loss with respect to canines. The box-auxillary were placed on canines to correct buccal torque loss of the canines (Figure 5.). Stage III, 0.021X0.028-inch archwires were then prepared, exactly as would be prescribed with tip-edge plus brackets. Auxiliary NiTi archwires (0.014 inch) were then threaded using a fine beak light wire plier through the deep tunnels, terminating in the gingivally-placed round molar tubes and the rectangular main archwires were fitted in the usual way (Figure 6.). After 5 months of root uprighting, appliances were removed. The post-treatment photograph, panoramic and cephalometric radiograph shows marked improvement in profile and facial aesthetics (Figure 7) and cephalometric summary shown in table 1. The pre and post treatment changes evaluated by superimposition of cephalometric tracings (Figure 8).
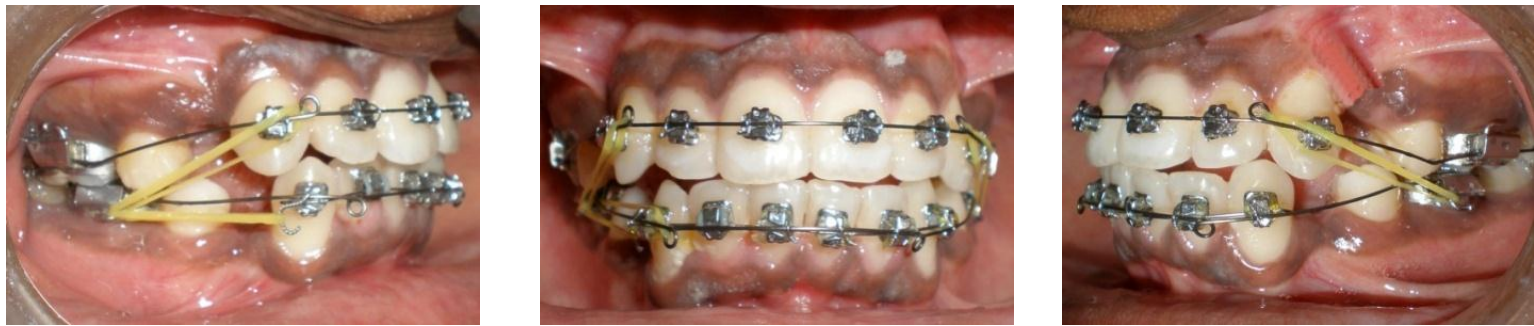

Fig 3. Stage I begins, using high-tensile 0.016-inch main archwires with bite-opening 'anchorage bends' and very light Class II elastic traction; 0.014-inch nickel titanium 'underarches' align the instanding incisors as the overjet and overbite reduce.
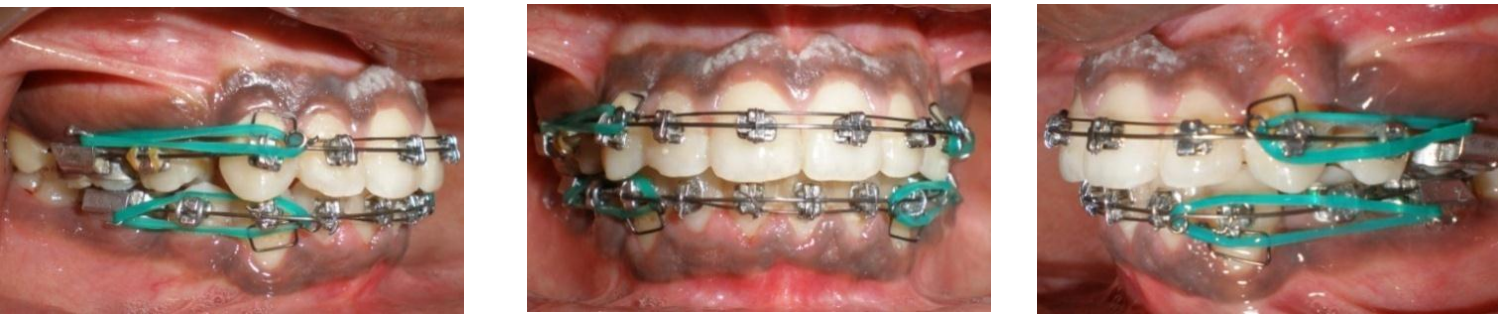

Fig 4. Five months into treatment, the remaining spaces was closed with 0.020 -inch stainless archwires and intramaxillary Elastomerics.
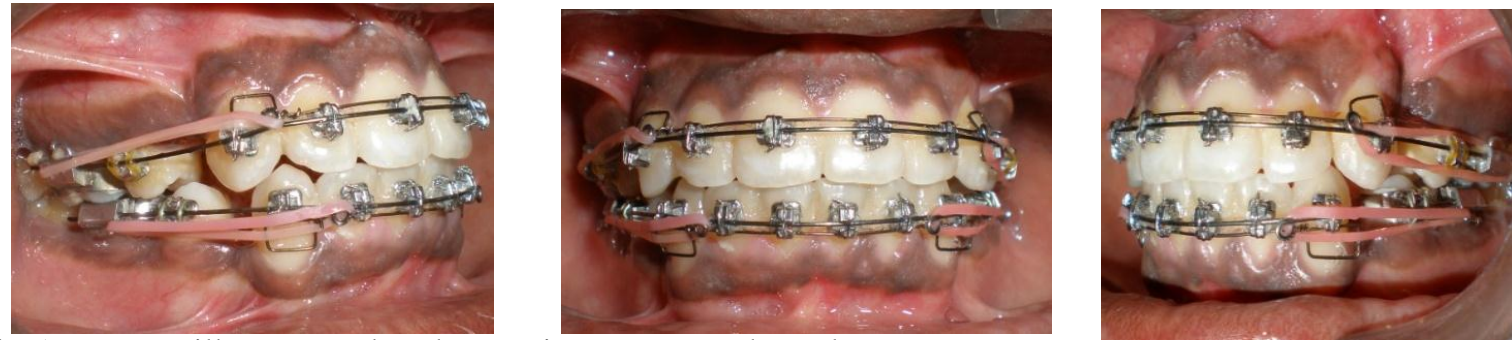

Fig 5. Box- auxillary were placed on canines to correct buccal root torque loss.
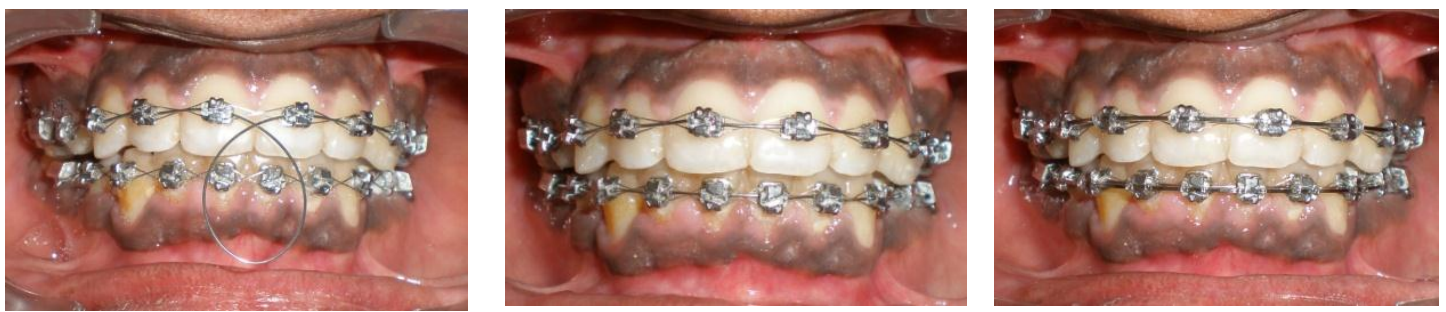
Experience with Contemporary Tip-Edge Plus Technique-A Case Report.

Figure 6 . Stage III begins by using auxiliary wire 0.014 -inch NiTi which was threaded through deep tunnel then $0.021 \times 0.028$-inch stainless steel main archwires placed.
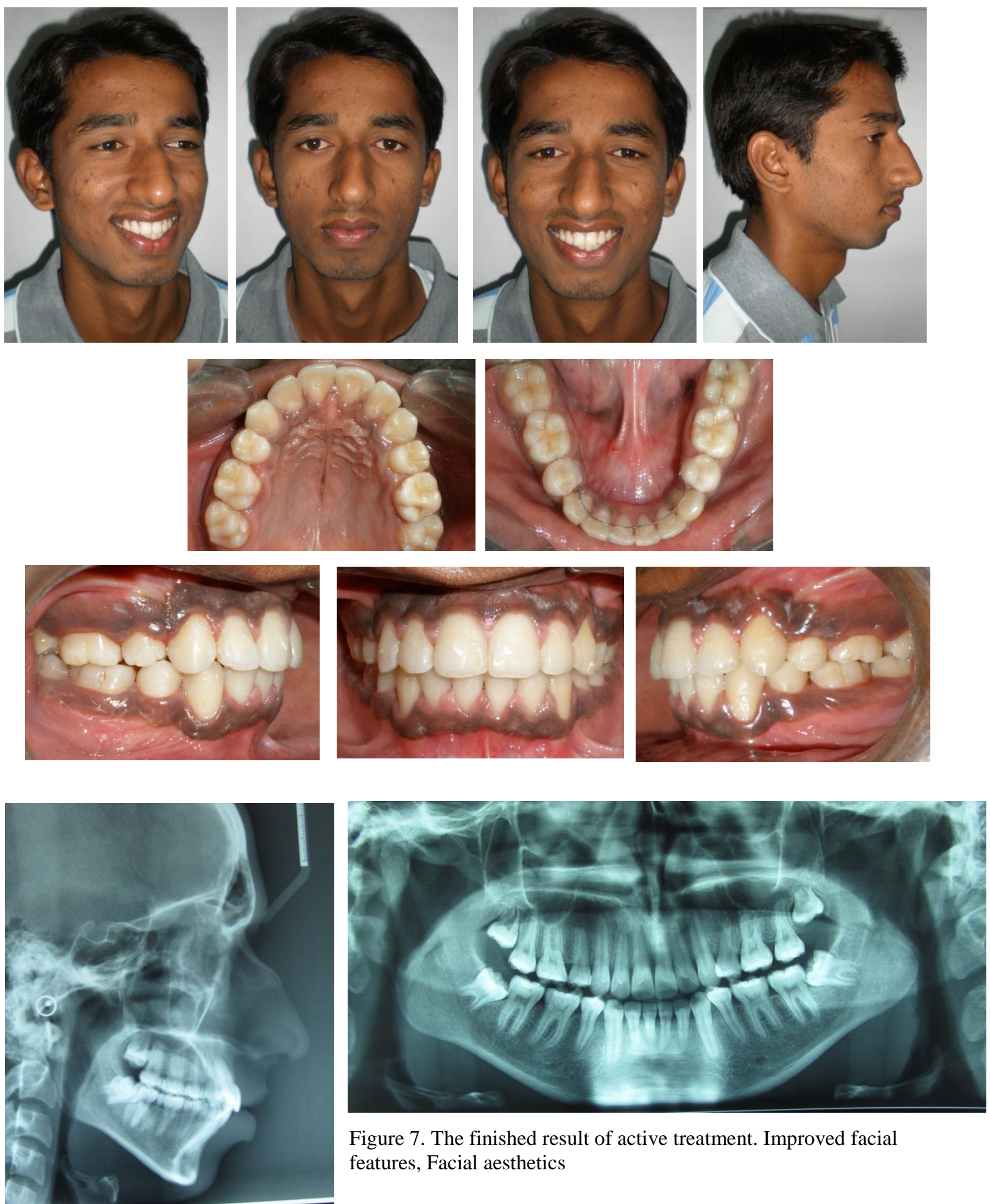

Figure 7. The finished result of active treatment. Improved facial features, Facial aesthetics 


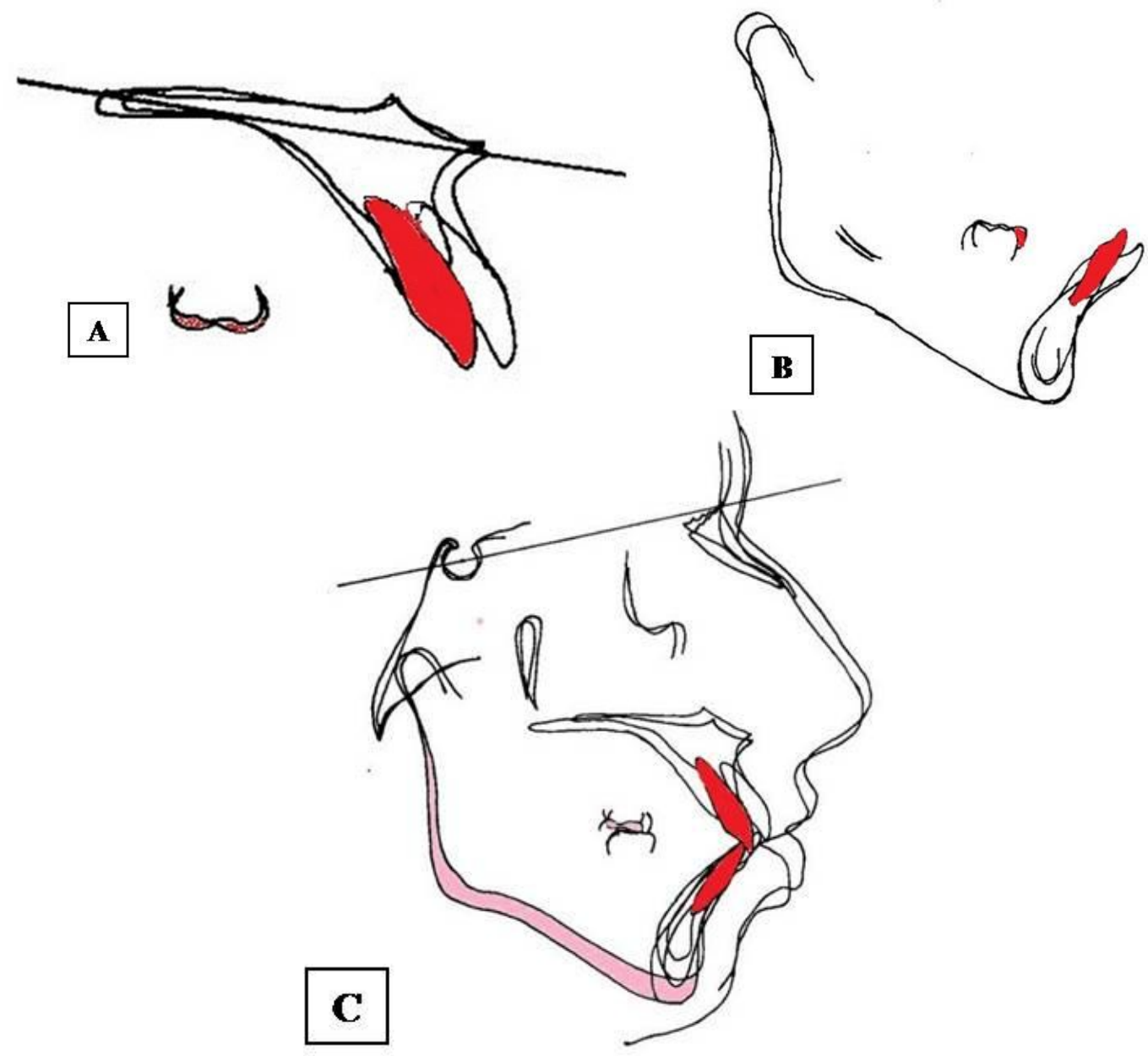

Figure 8 (A-C). Superimposition of cephalometric tracings of before and after treatment, A: Superimposition on palatal plane for maxillary dental changes. B: superimposition for mandibular dental changes by best fit method. C: overall superimposition on S-N plane registered at sella point

Table . 1 : Cephalometric Summary.

\begin{tabular}{|c|c|c|c|}
\hline CEPH & & & \\
\hline Parameters & Pre -Treatment & Post -Treatment & Difference \\
\hline SNA (degrees) & 84 & 81 & 3 \\
\hline SNB (degrees) & 79 & 78 & 2 \\
\hline "ANB (degrees) & 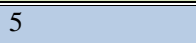 & 3 & 2 \\
\hline Wits analysis (mm) & 5 & 2 & 3 \\
\hline FMA(degrees) & 28 & 31 & 3 \\
\hline U1-SN (degrees) & 111 & 104 & $\overline{77}$ \\
\hline U1-A-Pog (mm) & 12 & 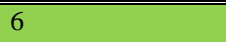 & 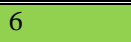 \\
\hline L1-MP (degrees) & 1110 & 96 & 14 \\
\hline LI to A-Pog (mm) & 9 & 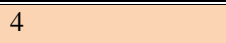 & 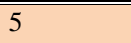 \\
\hline Nasolabial angle(degrees) & 98 & 102 & $\overline{44}$ \\
\hline Soft tissue facial angle(degrees) & 87 & 84 & 3 \\
\hline
\end{tabular}




\section{Conclusion:}

The aesthetics in Stage III are far superior and the appliance is easier to keep clean without auxiliary springs. It is also more comfortable, without the added labial profile of Side-Winders. Cooperation is improved because the patient is not tempted to interfere with the springs. The concept of 'Plus' is undeniably attractive, and it is likely to appeal to a wider user base than its predecessor.

\section{References:}

[1]. Jayne E. Harrison. Early Experiences with the Tip-Edge® Appliance. British Journal of Orthodontics 1998; 25:1-9.

[2]. Kesling PC. Expanding the horizons of the edgewise arch wire slot. Am J Orthod Dentofacial Orthop 1988; 94: $26-37$.

[3]. Kesling PC. Dynamics of the Tip-Edge bracket. Am J Orthod Dentofacial Orthop 1989; 96: 16-28.

[4]. Parkhouse RC. Current products and practice: Tip-Edge Plus. Journal of Orthodontics 2007;34:59-68

[5]. Parkhouse RC. Rectangular wire and third order torque: a new perspective. Am J Orthod Dentofacial Orthop 1998; 113: 421-30

[6]. Parkhouse RC. Tip-Edge Orthodontics. Edinburgh: Mosby,2003 